CPHT-S-751.1299

CTP-TAMU-47-99

CERN-TH/99-385

hep-ph/9912287

\title{
Looking For TeV-Scale Strings and Extra-Dimensions
}

\author{
E. Accomando ${ }^{a}$, I. Antoniadis ${ }^{b}$ and K. Benakli ${ }^{c}$ \\ ${ }^{a}$ Center for Theoretical Physics, Texas A\&3M University, College Station, TX77843-4242, USA \\ ${ }^{b}$ Centre de Physique Théorique, Ecole Polytechnique, 91128 Palaiseau, France \\ Unité mixte du CNRS et de l'EP, UMR 7644 \\ ${ }^{c}$ CERN Theory Division CH-1211, Genève 23, Switzerland
}

\begin{abstract}
In contrast to the old heterotic string case, the (weakly coupled) type I brane framework allows to have all, part or none of the standard model gauge group factors propagating in large extra-dimensions of $\mathrm{TeV}^{-1}$ size. We investigate the main experimental signatures of these possibilities, related to the production of Kaluza-Klein excitations of gluons and electroweak gauge bosons. A discovery through direct observation of resonances is possible only for compactification scales below $6 \mathrm{TeV}$. However effects due to exchange of virtual Kaluza-Klein excitations could be observed for higher scales. We find that LHC can probe compactification scales as high as $20 \mathrm{TeV}$ for excitations of gluons and 8-15 TeV for excitations of electroweak gauge bosons. Finally, in the case where no gauge boson feels the extra-dimension, we find that effective contact interactions due to massive string mode oscillations dominate those due to the exchange of Kaluza-Klein excitations of gravitons and could be used to obtain bounds on the string scale.
\end{abstract}

March 27, 2022 


\section{Introduction}

A lot of efforts have been devoted to understand possible patterns of new physics beyond the standard model. One of the most spectacular possibilities is the proposal of existence of large extra-dimensions compactified in the TeV range [1]. Such a scenario is easily realized if the fundamental string or quantum gravity scale is low [2]- [6].

Previous studies of the phenomenological implications of such extra-dimensions were focused on the simplest scenario where all standard model gauge bosons propagate in the same compact space [7]-[12]. Although this is the simplest scenario favored by gauge coupling unification [13, 14], it does not constitute the most general case. The different factors of the standard model gauge group may arise from branes of different kinds, extended in different compact directions. As a result, TeV-dimensions can be longitudinal along the world-volume of some branes and transverse to others.

A number of important questions arise in each of these cases: what are the experimental bounds on the size of such extra-dimensions? do these bounds allow the probe of extradimensions at future collider experiments? how can one distinguish the corresponding signals from other possible origin of new physics, such as models with new gauge bosons?

These questions can be addressed only in a well defined theoretical framework, where scenaria with large extra-dimensions can be realized consistently, such as perturbative type I string theory with fundamental scale $M_{s}$ of the order of a few TeV. . Our analysis concerns then longitudinal dimensions felt by gauge interactions, associated to compactification scales $R^{-1}$ somewhat smaller than the string scale, $R^{-1}<M_{s}$. The reason is that for string size dimensions, KK (Kaluza-Klein) excitations have masses comparable to string massive modes and their effects become indistinguishable. On the other hand, transverse dimensions larger than the string length are associated to superheavy open string winding modes which decouple at low energies; [2] they can be probed only through gravitational interactions associated to closed string KK modes.

Existing lower bounds on the compactification scale in the various cases of large extradimensions, come from indirect effects of KK modes in low or high energy measurements and are of the order of 2 to $4 \mathrm{TeV}$. These leave very little hope for production of many KK

\footnotetext{
${ }^{1}$ For notational simplicity, in this work we refer to type I any type $\mathrm{I}^{\prime}$ vacuum obtained by T-dualities from the original type I theory.

${ }^{2}$ This is true if bulk fields propagate always in more than two transverse dimensions, or if tadpoles cancel locally [3].
} 
excitations on-shell at LHC and none for other ongoing machines. In the most optimistic case, one will be able to observe just the first excitation, and thus, additional information or high precision will be necessary to bring evidence for its higher dimensional origin.

In section 2 we discuss the brane picture in models of Type I strings on orbifolds. This will allow us to illustrate different possibilities for localizing different parts of the observed particles on different branes. We obtain five classes of models that we study in the following sections. In section 3, we discuss the standard scenario where all the three gauge factors of the standard model feel the extra-dimension. New bounds on the upper value of the compactification scale accessible to LHC are obtained. These arise from the increase of

luminosity compared to 11] and the careful study of gluon and $W_{ \pm}$channels that have not received much of attention in the past (see [12]). In section 4, we discuss the collider signatures of the three other cases where only part of the standard model gauge group feels the large extra-dimension. Section 5 deals with the configuration where there are no longitudinal dimensions. We point out that in this case the effective contact interactions are dominated by effects due to tree-level exchange of massive string oscillation modes and the usually considered processes of virtual KK gravitons are sub-dominant. Finally section 6 summarizes our quantitative results.

\section{Bulk and boundary states within the brane picture}

In this work we are interested in collider experiments taking place at energies above the electroweak scale. Effects of the electroweak breaking can therefore be neglected and one can consider brane configurations that lead to unbroken $S U(3)_{c} \times S U(2)_{w} \times U(1)_{Y}$ gauge symmetry. Although no real realistic model has emerged yet from type I vacua [15, 6], we can discuss the main properties associated to different possible classes of such models.

In the generic case, the 3 group factors of the standard model may arise from different collections of coincident branes. If the space dimensionality $p$ of a set of branes ( $p$-branes) is bigger than 3 , there are $p-3$ longitudinal compact dimensions felt by the brane gauge interactions associated to the KK excitations with masses

$$
M_{n}^{2}=\frac{n^{2}}{R_{\|}^{2}}
$$

for integer $n$. Here, $R_{\|}$denotes generically the radii of longitudinal dimensions. The remaining $9-p$ transverse dimensions are felt only by gravity. The brane worldvolume fields 
have no KK excitations along these directions but (superheavy) winding modes with masses $|n| R_{\perp} M_{s}^{2}$, where $R_{\perp}$ denotes generically the radii of transverse dimensions. The size of compact dimensions is constrained by present observations to be less than $\mathrm{TeV}^{-1}$ for the longitudinal ones and less than millimeter for transverse. Below, we consider a particular dimension with a compactification scale in the $\mathrm{TeV}$ region, roughly an order of magnitude smaller than the string scale $R^{-1} \sim \mathcal{O}\left(10^{-1}\right) M_{s}$. The 3 sets of branes associated with the three standard model gauge group factors can then be either longitudinal or transverse with respect to this particular direction.

Since the coupling constant of the gauge group living on the longitudinal branes is reduced by the size of the large dimension $R M_{s}$ compared to those of the transverse branes, if $S U(3)$ has KK modes all three group factors must have. Otherwise it is difficult to reconcile the suppression of the strong coupling at the string scale with the observed reverse situation. As a result, there are 5 distinct cases that we denote $(l, l, l),(t, l, l),(t, l, t),(t, t, l)$ and $(t, t, t)$, where $l(t)$ refers to branes longitudinal (transverse) to the large dimension and the three positions in the brackets correspond to the 3 gauge group factors of the standard model $S U(3)_{c} \times S U(2)_{w} \times U(1)_{Y}$. In the first two cases, we will also comment on the anisotropic possibility of having more than one dimensions larger than the string length. We will ignore the possibility that a gauge factor is associated to a linear combination of $l$ and $t$ type of branes. Since the longitudinal component give rise to interactions suppressed by $R M_{s}$, this can be of importance only if the $t$ component is suppressed by a small mixing angle.

Similarly, there are three kinds of matter fields. Those which live on longitudinal or on transverse branes and those which arise as massless modes of open strings stretched between two different types of branes and live on the corresponding intersections. The first two types have KK and winding modes, respectively, along the large dimension, while the latter have no excitations and behave as the $Z_{2}$ twisted (boundary) states of heterotic strings on orbifolds. The boundary states couple to all KK-modes of gauge fields in the same way.f These couplings violate obviously momentum conservation in the compact direction and make all massive KK excitations unstable. If all quarks and leptons are of the first two kinds of matter fields living on branes, their interactions preserve the internal momentum and KK excitations (or windings) can be produced only in pairs and are stable. Naively, such a

\footnotetext{
${ }^{3}$ In contrast to the heterotic case open strings do not lead to $Z_{N}$ twisted matter with $N>2$.

${ }^{4}$ Actually, when one is restricted to physical states with positive KK momentum $n$ (see eq. (1)), the coupling of massive excitations is enhanced by a factor $\sqrt{2}$ relative to the lowest (massless) one.
} 
possibility is excluded, for instance from the non-observation of stable excitations bounded to atomes. However, this assumes their production during the early history of the universe which might be suppressed if the "maximal" temperature is very low as suggested in [16. In the following we ignore this possibility and assume that all matter fields are localized in the large dimension, with the exception of the $(t, t, t)$ case which we treat in section 5 .

In addition to the KK excitations of massless modes there might be massive modes with different quantum numbers. Their corresponding massless modes are projected out since they transform non-trivially under the orientifold projection that leads to chiral spectrum. These odd states can be new gauge bosons that enlarge the gauge symmetry or extra fermions and scalars. They can decay only to localized matter fields, but their coupling vanishes to lowest order. Non-vanishing couplings can be obtained by taking derivatives along the extradimension and are therefore suppressed by powers of $R M_{s}$. For instance, the lowest order of such operators (in non-supersymmetric models) involves three scalars one of which is odd and has a derivative acting on it. These states are in general model dependent and will not be discussed here.

\section{The "standard" $(l, l, l)$ scenario:}

In the $(l, l, l)$ scenario all gauge bosons of the standard model $S U(3)_{c} \times S U(2)_{w} \times U(1)_{Y}$ have KK excitations. Because matter fields are localized, their interactions do not preserve the momenta in the extra-dimension and single KK excitations can be produced. This means for example that QCD processes $q \bar{q} \rightarrow G^{(n)}$ with $q$ representing quarks and $G^{(n)}$ massive $\mathrm{KK}$ excitations of gluons are allowed. In contrast processes such as $G G \rightarrow G^{(n)}$ are forbidden as gauge boson interactions conserve the internal momenta. The exchange of virtual KK modes leads to effects in low energy processes that are constrained by fits to high precision data [9, 10]. For instance, fit of measured values of $M_{W}, \Gamma_{l l}$ and $\Gamma_{\text {had }}$ lead to $R^{-1} \gtrsim 3.5 \mathrm{TeV}$. Inclusion of $Q_{W}$ measurement, which does not give a good agreement with the standard model itself, raises the bound to $R^{-1} \gtrsim 3.9 \mathrm{TeV}$ [10].

For the LHC experiment, this scenario predicts that new resonances in three different channels $l^{+} l^{-}, l^{ \pm} \nu$ and dijets could be observed at the same mass if the compactification scale is low enough. The resonances due to the $S U(3)$ gluon excitations have quite large widths due to the "strong" coupling value. They are thus spread and difficult to detect already for compactification scales of the order of $5 \mathrm{TeV}$. In figure 1 we show the shape of 
a typical expected signal for the production of a gluon excitation for $R^{-1}=4$ and $5 \mathrm{TeV}$ at the LHC (assuming $\sqrt{s}=14 \mathrm{TeV}$ and $L=100 \mathrm{fb}^{-1}$ ). Our results have been obtained using the CTEQ4L structure funcions [17]. We have also included the multiplicative Kfactor [18], taken to be $K=1.1$, and summed over all jets, top excluded. Moreover, we have implemented a rapidity cut, $|\eta| \leq 0.5$, on both jets and required the invariant mass to be $M_{j j^{\prime}} \geq 2 \mathrm{TeV}$.

The strength of the coupling constant of gluon excitations implies on the other hand a large excess in the total number of events from the QCD expected ones. We found that looking for excess of dijet events could be the most efficient channel to constrain the size of extra-dimensions. In figure 2, we present the effect of these gluonic KK states on the total number of dijet events. The significance, given by the ratio $\left|N_{T}-N_{S M}\right| / \sqrt{N_{S M}}$ where $N_{T}$ is the total number of events and $N_{S M}$ is the SM background, indicates that LHC could probe in this hadronic channel values of the compactification scale up to about $20 \mathrm{TeV}$. This becomes of order $15 \mathrm{TeV}$ for a lower luminosity of $L=10 \mathrm{fb}^{-1}$ used in [11]. The Tevatron run II with luminosity of $L=20 \mathrm{fb}^{-1}$ will probe compactification scales of order $4 \mathrm{TeV}$.

The KK-excitations $W_{ \pm}^{(n)}$ of the $\mathrm{W}$ bosons could show up in clean final states consisting of one charged lepton and missing energy $\left(\nu_{l} l\right.$ with $\left.l=e, \mu\right)$. The differential cross-section in the transverse mass has the shape illustrated for $R^{-1}=4,5$ and $6 \mathrm{TeV}$ in figure 3 . We have required the charged lepton to be in the central region, $|\eta| \leq 1$. This cut reduces the SM background by a factor of about $50 \%$, while decreasing the pure signal only by $35 \%$ due to the large masses considered. The discovery limit of such a resonance is around $6 \mathrm{TeV}$.

Approximately the same values of compactification scales $\lesssim 6 \mathrm{TeV}$ could be discovered through the observation of a resonance in the $l^{+} l^{-}$final state (with $l=e, \mu$ ). The interference in the overlap of the resonances $\gamma^{(n)}$ of the photon and $Z^{(n)}$ leads to an a priori distinctive deep as noticed in [11, 12]. The shape of the resonance is quite different from the one of a Bright-Wigner due to the excitation of only one gauge boson, as can be seen in figure 3 .

In a way similar to the case of excitations of gluons, the exchange of virtual KK excitations $W_{ \pm}^{(n)}, \gamma^{(n)}$ and $Z^{(n)}$ lead to deviations from the standard model expectation in the number of total events. These allow to probe higher compactification scales. Figure 4 shows that at $95 \%$ confidence level LHC could exclude values of compactification scales up to 12, 14 and $15 \mathrm{TeV}$ from the photon $+Z, W_{ \pm}$and combined channels, respectively. Here, for the dilepton final state, we have required one lepton to be in the central region, $\left|\eta_{l}\right| \leq 1$, the other one having a looser cut $\left|\eta_{l^{\prime}}\right| \leq 2.4$. This gives about the same acceptance as above. Moreover, 
we have chosen a $400 \mathrm{GeV}$ lower bound on the transverse and invariant mass, in order to optimize the significance.

\section{The cases $(t, l, l),(t, l, t)$ and $(t, t, l)$ :}

The difference between the $(t, l, l)$ and the $(l, l, l)$ scenario is that in the former the gluons do not feel the extra-dimensions. Only the KK excitations $W_{ \pm}^{(n)}, \gamma^{(n)}$ and $Z^{(n)}$ are present and lead to the same effects as for the $(l, l, l)$ case. The limits on the extra-dimensions follow from the previous section: $6 \mathrm{TeV}$ for discovery and $15 \mathrm{TeV}$ for the exclusion bounds.

In the $(t, l, t)$ case, only the $S U(2)$ factor arises from a set of branes longitudinal to the $\mathrm{TeV}$-scale extra-dimension. As all the states charged under $U(1)_{Y}$ are localized in this case, one has no more the freedom to choose the Higgs to be localized or not as it was for the cases $(l, l, l)$ and $(t, l, l)$ but only the first possibility exists. The massive modes are KK excitations of $W_{ \pm}$and $W_{3}$. The latter will lead to a deficit in $l^{+} l^{-}$half of the corresponding one in the $l^{ \pm} \nu$ channel (as two gauge bosons contribute here). Moreover, the forward-backward asymmetry is maximal. The limits on this scenario arise from exchange of $W_{ \pm}$and are again $6 \mathrm{TeV}$ for discovery and $14 \mathrm{TeV}$ for the exclusion bounds.

The third case is the one of $(t, t, l)$ channel where only $U(1)_{Y}$ feels the extra-dimension. Similarly to the previous case, the Higgs doublets have to be identified with localized states. In this case the limits are weaker, the exclusion bound is in fact around $8 \mathrm{TeV}$, as can be seen in figure 6.

Finally, let us comment about some of the possibilities that we neglected. In the scenaria discussed above, we have assumed that only one extra-dimension is large and longitudinal to some sets of branes. In the $(t, l, l)$ and the $(l, l, l)$ cases one could imagine that the different gauge factors arise from different sets of branes feeling different dimensions. In this case, the $\gamma^{(n)}+Z^{(n)}$ resonances split into resonances of $W^{3}$ and $U(1)_{Y}$ located at different masses. Another possibility to notice is that the case where part of $U(1)_{Y}$ is $t$ and part is $l$, one would observe a signal which is hard to distinguish from a generic extra $U(1)^{\prime}$. A good statistic would be needed to distinguish the deviation in the tail of the resonance as being due to effects additional to those of the $U(1)^{\prime}$ itself. 


\section{Limit on the string scale:}

Finally we discuss the case $(t, t, t)$ where all the extra-dimensions are transverse to the 3-brane where the standard model lives. In this case standard model particles have no KK excitations and the main experimental signals are due either to gravitational effects associated to closed strings propagating in the ten-dimensional bulk or to the exchange of massive string oscillation modes. The gravitational effects were extensively studied in the litterature based on graviton exchange in the effective field theory [19, 20, 21]. In the context of Type I string models, these effects are part of the one-loop diagram of open strings (annulus) that can be seen as closed strings exchanged in the transverse channel (cylinder). However these are subdominant, $\mathcal{O}\left(g_{s}^{2}\right)$ with $g_{s}$ the string coupling, compared to the tree level exchange of open string oscillation modes, which are of order $\mathcal{O}\left(g_{s}\right)$ 甲.

Indeed, the exchange of virtual gravitons is described in the effective field theory by an amplitude of the form $\mathcal{S}(s) \mathcal{T}$ with [19]:

$$
\mathcal{T}=T_{\mu \nu} T^{\mu \nu}-\frac{1}{1+d_{\perp}} T_{\mu}^{\mu} T_{\nu}^{\nu}
$$

and

$$
\mathcal{S}=\frac{1}{M_{p}^{2}} \sum_{n} \frac{1}{s-\frac{\vec{n}^{2}}{R_{\perp}^{2}}}
$$

where $T^{\mu \nu}$ is the energy momentum tensor and $s$ the center of mass energy. The sum in $\mathcal{S}$ is divergent for a number $d_{\perp}>1$ of transverse dimensions. From the string theory point of view this corresponds to an ultraviolet one-loop divergence cut-off appropriately by the string scale. The final result is finite and model (compactification) dependent. A phenomenological approach followed in [7, 20, 21] is to estimate $\mathcal{S}$ as

$$
\mathcal{S}=g_{s}^{2} \frac{A}{M_{s}^{4}}
$$

where $A=\log \frac{M_{s}^{2}}{s}$ for $d_{\perp}=2$ and $A=\frac{2}{d_{\perp}-2}$ for $d_{\perp}>2$. The coupling constant factor $g_{s}^{2}$ arises from the Type I relation $M_{p}^{2}=M_{s}^{2}\left(M_{s} R_{\perp}\right)^{d_{\perp}} / g_{s}^{2}$ and the fact that the divergent sum is cut-off at the string scale $M_{s}$.

On the other hand, the tree-level open string four-point amplitude is:

$$
\mathcal{A}(1,2,3,4)=\frac{1}{M_{s}^{2}} g_{s} \frac{\Gamma\left(1-s / M_{s}^{2}\right) \Gamma\left(1-t / M_{s}^{2}\right)}{\Gamma\left(1-s / M_{s}^{2}-t / M_{s}^{2}\right)} K(1,2,3,4)+[s \rightarrow t]+[s \rightarrow u]
$$

\footnotetext{
${ }^{5}$ This observation is also made recently by M. Peskin in [22.
} 
where $K$ contains kinematic factors as well as gauge indices (Chan-Paton factors) [23]. We have introduced the Mandelstam kinematic invariants:

$$
s=-\left(p_{1}+p_{2}\right)^{2} \quad t=-\left(p_{1}+p_{3}\right)^{2} \quad u=-\left(p_{1}+p_{4}\right)^{2}
$$

with $p_{i}$ the momentum of state $i$. Expanding the Gamma-functions at low energies in powers of $s / M_{s}^{2}$, and summing over the three permutations, one finds for the 4-fermion amplitude:

$$
\mathcal{A}(1,2,3,4) \simeq g_{s} \frac{\mathcal{A}_{s}}{s}\left(1+\frac{\pi^{2}}{12} \frac{s^{2}}{M_{s}^{4}}\right)+g_{s} \frac{\mathcal{A}_{t}}{t}\left(1+\frac{\pi^{2}}{12} \frac{t^{2}}{M_{s}^{4}}\right)+g_{s} \frac{\mathcal{A}_{u}}{u}\left(1+\frac{\pi^{2}}{12} \frac{u^{2}}{M_{s}^{4}}\right) .
$$

Note that the leading contribution $g_{s}\left(\frac{\mathcal{A}_{s}}{s}+\frac{\mathcal{A}_{t}}{t}+\frac{\mathcal{A}_{u}}{u}\right)$ reproduces the point-like particle result. In $\mathcal{A}_{s}$ we have absorbed the kinematical as well as the trace on Chan-Paton trace factors:

$$
\mathcal{A}_{s}=\operatorname{Tr}\left(\lambda^{1} \lambda^{2} \lambda^{3} \lambda^{4}\right) \bar{u}_{1} \gamma^{\mu} u_{2} \bar{u}_{3} \gamma_{\mu} u_{4}
$$

where $\lambda^{i}$ are the Chan-Paton factors, $u_{i}$ are the associated spinors and we dropped numerical factors. The $\mathcal{A}_{t}$ and $\mathcal{A}_{u}$ are obtained by replacing the cyclic order 1234 by 1342 and 1423 respectively. For example, in the usual Bha-Bha scattering, $\mathcal{A}_{u}$ vanishes due to the traces on Chan-Paton factors and the two terms with poles in $s$ and $t$ channels remain. The next terms describe effective contact interactions. A comparison with eq. (4) shows that they are enhanced by a string-loop factor $g_{s}^{-1}$ with respect to the field theory estimate for KK graviton exchanges. Although the precise value of $g_{s}$ requires a detailed analysis of threshold corrections, for a rough estimate one could take $g_{s} \simeq \alpha \sim 1 / 25$, that implies an enhancement by an order of magnitude.

As a result, one can not get reliable experimental bounds on the string scale just based on fits of differential cross-sections with field theoretical estimates for the contribution of graviton KK exchange, but should use processes with missing energy due to emission of "onshell" gravitons in the bulk [19]. Alternatively, the four-point contact interactions analyzed above may also be used to provide direct bounds on the string scale. Note however that these interactions are in general model dependent.

Above we have considered the case of 4 -fermion interactions which receive lowest order contributions at tree-level from massless fields. However, one could also consider processes for which the dominant lowest order contributions arise from exchange of massive open string oscillator modes. Such an example is the four-point amplitude of abelian gauge fields, which gives rise to leading contact interactions encoded in the Born-Infeld action [23]. 


\section{Conclusions}

We have described various possible models allowed by the Type I picture of the brane-world scenario. Assuming that matter fermions are localized in some large $\mathrm{TeV}$ extra-dimension and that there is no inverse hierarchy of the tree-level gauge couplings compared to the observed (loop-corrected) ones, we found 5 classes of models, denoted as $(l, l, l),(t, l, l)$, $(t, l, t),(t, t, l)$ and $(t, t, t)$. For these models we have attempted to answer the questions raised in the introduction. What are the experimental bounds on the size of such extradimensions? Lower bounds on the compactification scale from low energy measurements are of the order of 3 to $4 \mathrm{TeV}$.

Do these bounds allow the probe of extra-dimensions at future collider experiments? We have found that new resonances can be discovered at LHC for scales as high as around $6 \mathrm{TeV}$ for all the four cases $(l, l, l),(t, l, l),(t, l, t)$ and $(t, t, l)$. This implies that only a small window of 3 - 4 to $6 \mathrm{TeV}$ for the compactification scale is left. Improvement of low energy precision bounds from existing experiments (LEP, Tevatron...) are thus of great importance as they may allow to narrow further this window. Higher scales can be probed through indirect effects of exchanges of virtual KK excitations. We found that the most efficient channel is through $\mathrm{KK}$ excitations of gluons which give sensitivity up to $R^{-1} \lesssim 20 \mathrm{TeV}$. The $W_{ \pm}$, photon $+Z$ and $U(1)_{Y}$ boson allow to probe values $R^{-1} \lesssim 14,12$ and $8 \mathrm{TeV}$, respectively.

How can one distinguish the corresponding signals from other possible origin of new physics, such as models with new gauge bosons? The discussion on this issue follows the one for the $(t, l, l)$ case in [11, 12]. We have pointed out that scenaria $(l, l, l),(t, l, l)$ and $(t, l, t)$ predict the existence of resonances in three or two final channels. As for the $(t, l, l)$ case in [11, 12, we have pointed out that models $(l, l, l)$ and $(t, l, t)$ predict the existence of resonances in three and two final channels, respectively. They are located at the same value of energy. This property is not shared by most of other new gauge boson models. Moreover, the heights and widths of the resonances are directly related to those of standard model gauge bosons in the corresponding channels. Also, in the case of excitations of the photon $+Z$ a deep due to the interference between the two bosons should be observed just before the resonance.

For the last case $(t, t, t)$, we pointed out that there are open-string tree-level contact terms that are dominant with respect of those induced by the exchange of KK excitations of gravitons. Analysis of these terms should allow in principle to get stronger bounds on the 
string scale. The precise coefficients, however, of the higher-dimensional effective operators are in general model (compactification) dependent and further investigation is needed.

We wish to thank T. Kamon and Y. Oz for discussions at various stages of the work. KB wishes also to thank A. Delgado, A. Pomarol, M. Quiros and J. Lykken for discussion. This work is supported in part by the EEC under TMR contract ERBFMRX-CT96-0090 and in part by NSF grant No. PHY-9722090.

\section{References}

[1] I. Antoniadis, Phys. Lett. B246 (1990) 377

[2] N. Arkani-Hamed, S. Dimopoulos and G. Dvali, Phys. Lett. B429 (1998) 263; I. Antoniadis, N. Arkani-Hamed, S. Dimopoulos and G. Dvali, Phys. Lett. B436 (1998) 257 ;

[3] I. Antoniadis and C. Bachas, Phys. Lett. B450 (1999) 83.

[4] J.D. Lykken, Phys. Rev. D54 (1996) 3693; I. Antoniadis and B. Pioline, Nucl. Phys. B550 (1999) 41; K. Benakli and Y. Oz, hep-th/9910090.

[5] K. Benakli, Phys. Rev. D60 (1999) 104002; C.P. Burgess, L.E. Ibáñez and F. Quevedo, Phys. Lett. B447 (1999) 257.

[6] G. Shiu and S.-H.H. Tye, Phys. Rev. D58 (1998) 106007; Z. Kakushadze and S.H.H. Tye, Nucl. Phys. B548 (1999) 180; L.E. Ibáñez, C. Muñoz and S. Rigolin, hepph/9812397; G. Aldazabal, L.E. Ibáñez and F. Quevedo, hep-th/9909172.

[7] I. Antoniadis and K. Benakli, Phys. Lett. B326 (1994) 69.

[8] I. Antoniadis, C. Muñoz and M. Quirós, Nucl. Phys. B397 (1993) 515; I. Antoniadis, K. Benakli and M. Quirós, Phys. Lett. B331 (1994) 313; K. Benakli, Phys. Lett. B386 (1996) 106; I. Antoniadis and M. Quirós, Phys. Lett. B392 (1997) 61; I. Antoniadis, S. Dimopoulos and G. Dvali, Nucl. Phys. B516 (1998) 70; A. Pomarol and M. Quirós, Phys. Lett. B438 (1998) 225; I. Antoniadis, S. Dimopoulos, A. Pomarol and M. Quirós, Nucl. Phys. B544 (1999) 503; A. Delgado, A. Pomarol and M. Quirós, hep-ph/9812489. 
[9] P. Nath and M. Yamaguchi, hep-ph/9902323 and hep-ph/9903298; M. Masip and A. Pomarol, hep-ph/9902467; W.J. Marciano, Phys. Rev. D60 (1999) 093006; A. Strumia, hep-ph/9906266; R. Casalbuoni, S. De Curtis, D. Dominici and R. Gatto, hepph/9907355; C.D. Carone, hep-ph/9907362.

[10] A. Delgado, A. Pomarol and M. Quirós, hep-ph/9911252

[11] I. Antoniadis, K. Benakli and M. Quiros, Phys. Lett. B460 (1999) 176.

[12] P. Nath, Y. Yamada and M. Yamaguchi, hep-ph/9905415; T.G. Rizzo and J.D. Wells, hep-ph/9906234; T.G. Rizzo, hep-ph/9909232.

[13] K.R. Dienes, E. Dudas and T. Gherghetta, Phys. Lett. B436 (1998) 55 and Nucl. Phys. B537 (1999) 47; D. Ghilencea and G.G. Ross, Phys. Lett. B442 (1998) 165; Z. Kakushadze, hep-th/9811193; C.D. Carone, hep-ph/9902407; A. Delgado and M. Quirós, hep-ph/9903400; P. Frampton and A. Rasin, hep-ph/9903479; A. PérezLorenzana and R.N. Mohapatra, hep-ph/9904504; Z. Kakushadze and T.R. Taylor, hep-th/9905137; K. Huitu and T. Kobayashi, hep-ph/9906431; Y. Abe, C. Hattori, M. Ito, M. Matsunaga and T. Matsuoka, hep-ph/9902472; T. Kobayashi, J. Kubo and G. Zoupanos, hep-ph/9812221; H.-C. Cheng, B. A. Dobrescu and C.T. Hill, hepph/9906327.

[14] C. Bachas, JHEP 9811 (1998) 23; I. Antoniadis, C. Bachas and E. Dudas, hepth/9906039; N. Arkani-Hamed, S. Dimopoulos and J. March-Russell, hep-th/9908146.

[15] A. Sagnotti, hep-th/9302099; C. Angelantonj, M. Bianchi, G. Pradisi, A. Sagnotti and Ya.S. Stanev, Phys. Lett. B385 (1996) 96; Z. Kakushadze and G. Shiu，Phys. Rev. D56 (1997) 3686 and Nucl. Phys. B520 (1998) 75; M. Berkooz and R. Leigh, Nucl. Phys. B483 (1997) 187; G. Zwart, hep-th/9708040; Z. Kakushadze, Nucl. Phys. B512 (1998) 221; G. Aldazabal, A. Font, L.E. Ibáñez and G. Violero, hep-th/9804026; J. Lykken, E. Poppitz and S.P. Trivedi, Nucl. Phys. B543 (1998) 105; G. Aldazabal, D. Badagnani, L.E. Ibáñez and A. Uranga, hep-th/9904071; C. Angelantonj, I. Antoniadis, G. D'Appollonio, E. Dudas and A. Sagnotti, hep-th/9911081.

[16] N. Arkani-Hamed, S. Dimopoulos and G. Dvali, Phys. Rev. D59 (1999) 086004; K. Benakli and S. Davidson, Phys. Rev. D60 (1999) 025004; L.J. Hall and D. Smith, Phys. Rev. D60 (1999) 085008. 
[17] H.J. Lai, J. Huston, S. Kuhlmann, F. Olness, J. Owens, D. Soper, W.K. Tung and H. Weerts, Phys. Rev. D55 (1997) 1280.

[18] R. Hamberg, W.L. van Neerven and T. Matsuura, Nucl. Phys. B359 (1991) 343.

[19] G.F. Giudice, R. Rattazzi and J.D. Wells, hep-th/9811292; E.A. Mirabelli, M. Perelstein and M.E. Peskin, hep-ph/9811337.

[20] T. Han, J. Lykken and R.-J. Zhang, hep-ph/9811350.

[21] See, for example: J.L. Hewett, hep-ph/9811356; E. Dudas and J. Mourad, hepth/9911019; T.G. Rizzo, Phys. Rev. D60 (1999) 115010; P. Mathews, S. Raychaudhuri and K. Sridhar, hep-ph/9811501; K. Cheung, W.-Y. Keung, hep-ph/9903294; K. Cheung, G. Landsberg, hep-ph/9909218; H. Davoudiasl, hep-ph/9904425; P. Mathews, P. Poulose and K. Sridhar, hep-ph/9905395; H.-W. Yu, L.H. Ford, gr-qc/9907037; T. Han, D. Rainwater and D. Zeppenfeld, hep-ph/9905423; K. Agashe, N.G. Deshpande, hep/9902263; M.L. Graesser, hep-ph/9902310; D.Atwood, S. Bar-Shalom, A. Soni, hep-ph/9903538; hep-ph/9906400; D. Bourilkov, hep-ph/9907380; M. Besancon, hep-ph/9909364; see also: S. Nussinov and R. Shrock, Phys. Rev. D59 (1999) 105002; G. Shiu, R. Shrock and S.H. H. Tye, hep-ph/9904262; T. Banks, Michael Dine, Ann Nelson, hep-th/9903019; E. Halyo, hep-ph/9904432; A. Donini and S. Rigolin, hepph/9901443; J. Lykken and S. Nandi, hep-ph/9908505; B. Grzadkowski and J.F. Gunion, hep-ph/9910456.

[22] M. Peskin, talk at ITP Santa Barbara, http://www.itp.ucsb.edu/online/susy_c99/. [23] See, for example: J. Polchinski, String theory, Cambridge press, 1998. 


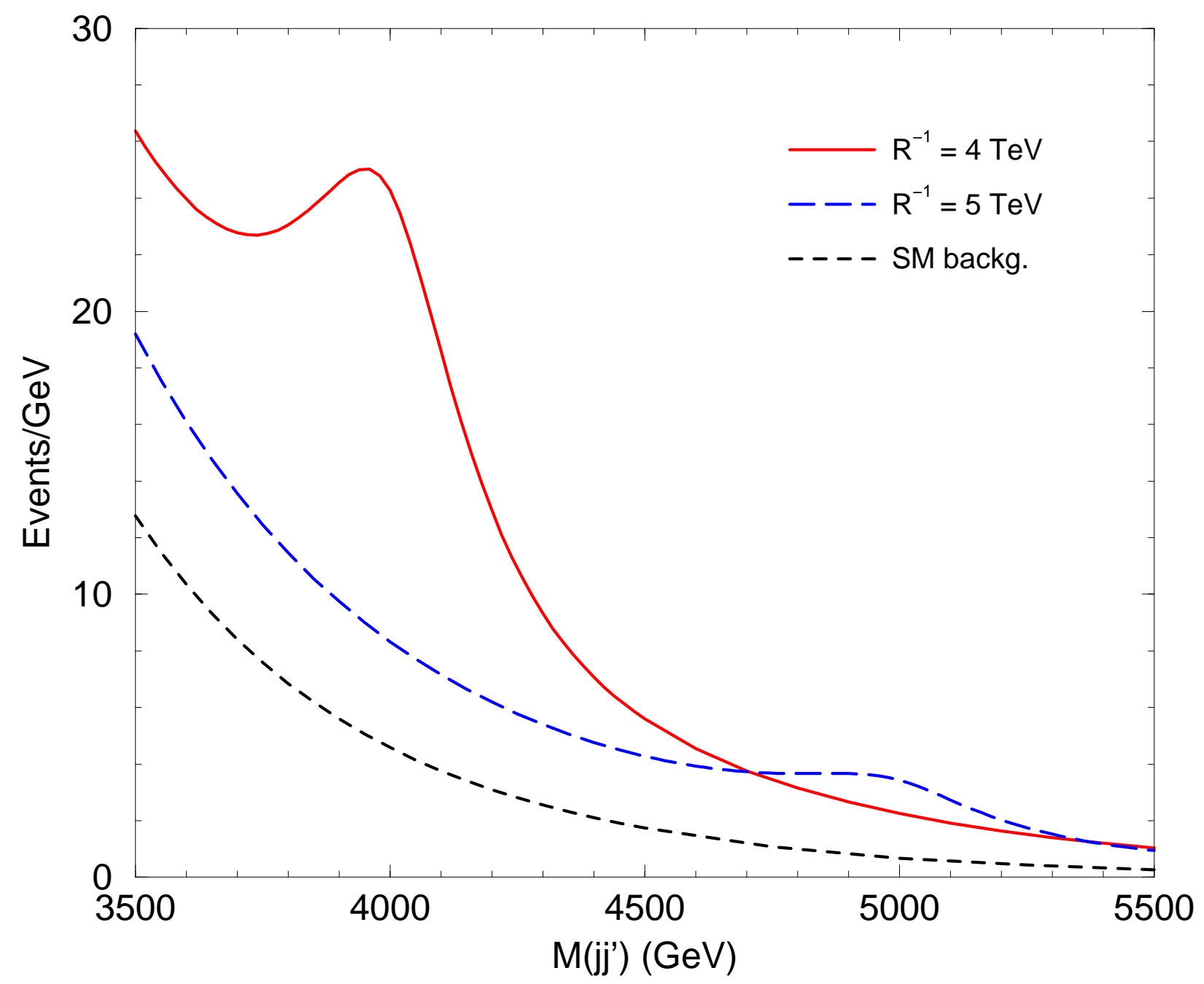

Figure 1: First resonances in the LHC experiment due to a KK excitation of gluon for one extra-dimension at 4 and $5 \mathrm{TeV}$. 


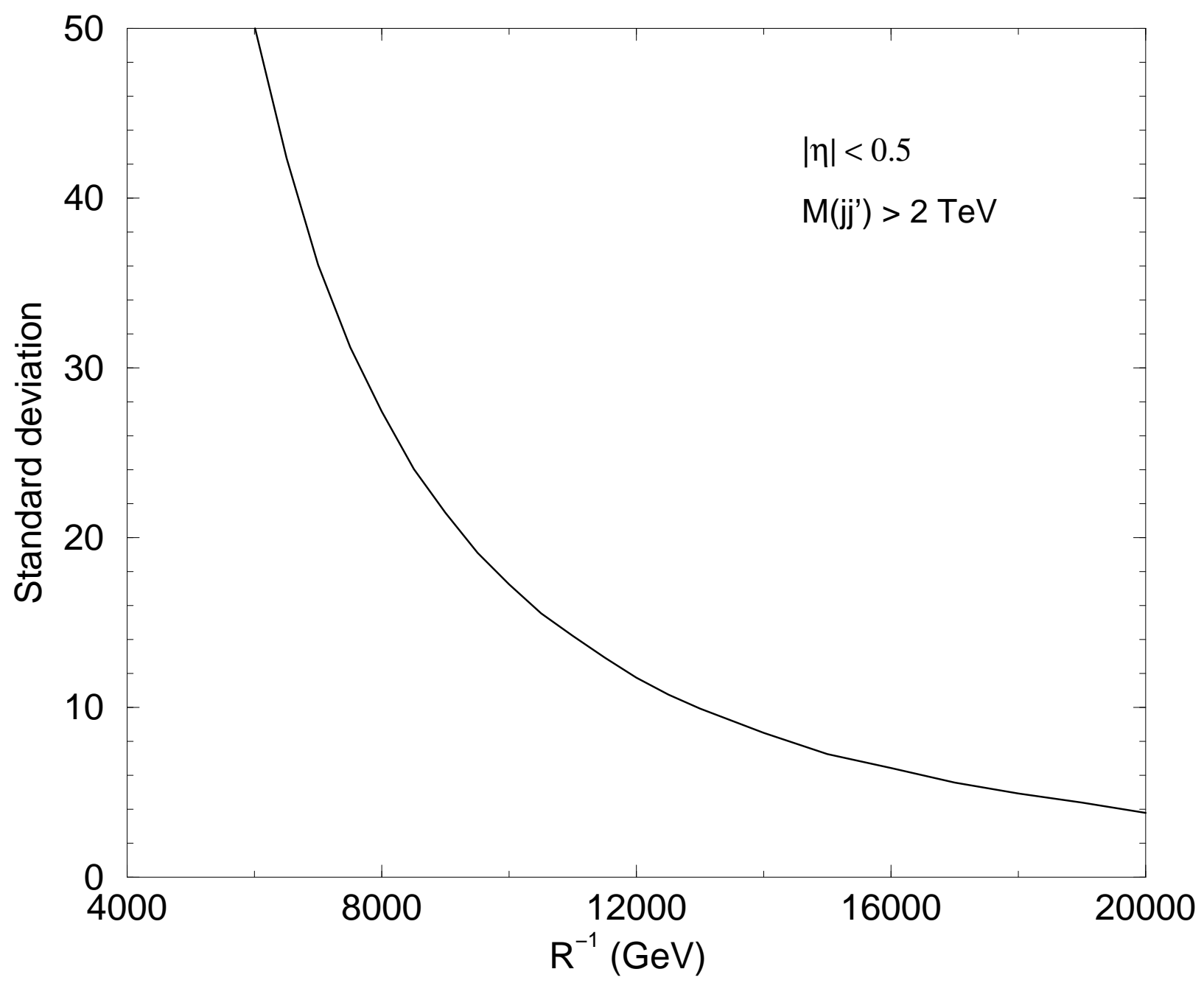

Figure 2: Number of standard deviation in number of observerd dijets from the expected standard model value, due to the presence of a TeV-scale extra-dimension of compactification radius $R$. 


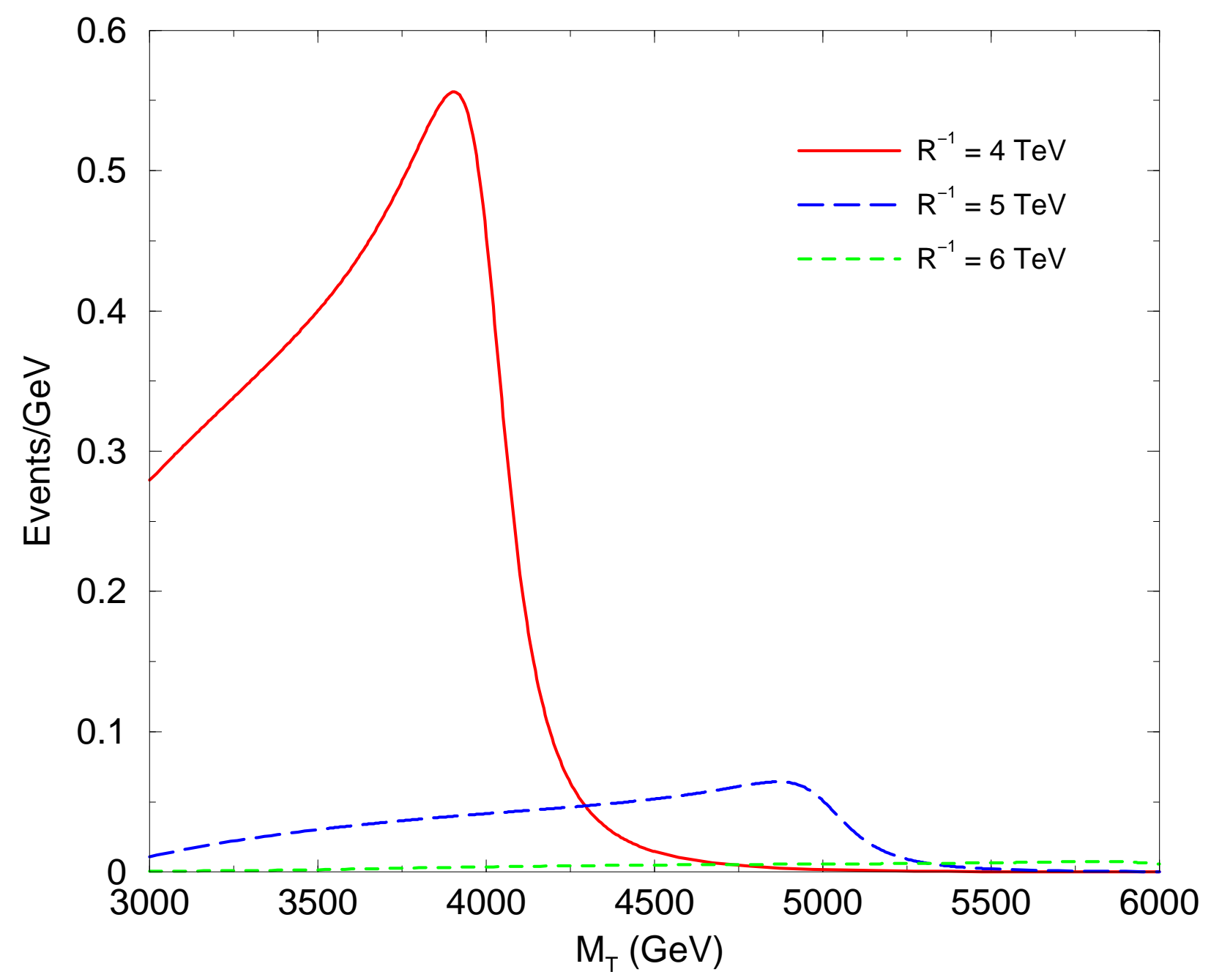

Figure 3: First resonances in the LHC experiment due to a KK excitation of $W_{ \pm}^{(n)}$ for one extra-dimension at 4, 5 and $6 \mathrm{TeV}$. We plot the differential cross section as function of the transverse mass for the $W s$. 


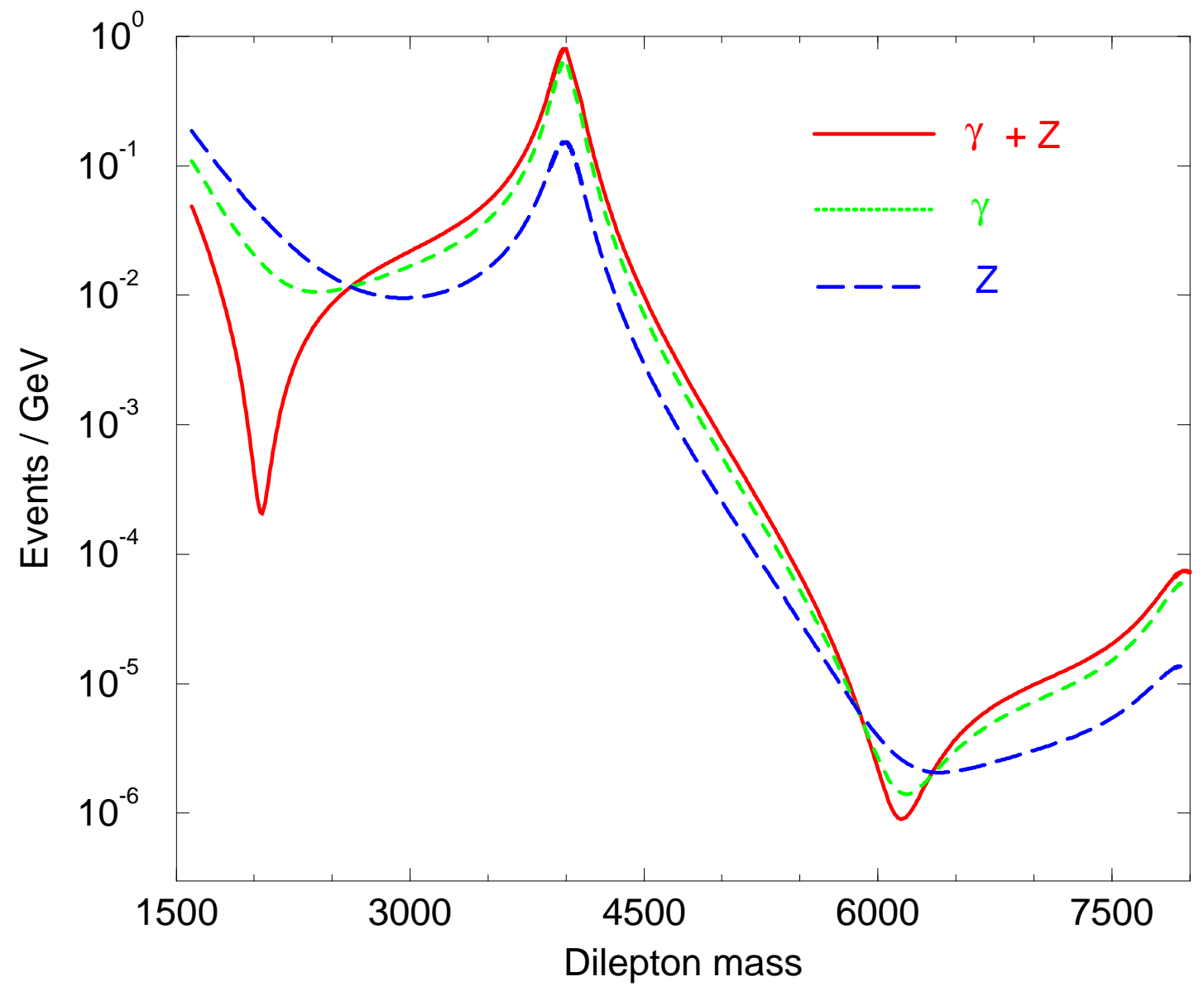

Figure 4: First resonances in the $L H C$ experiment due to a $K K$ excitation of photon and $Z$ for one extra-dimension at $4 \mathrm{TeV}$. From highest to lowest: excitation of photon $+Z$, photon and $Z$ boson. 


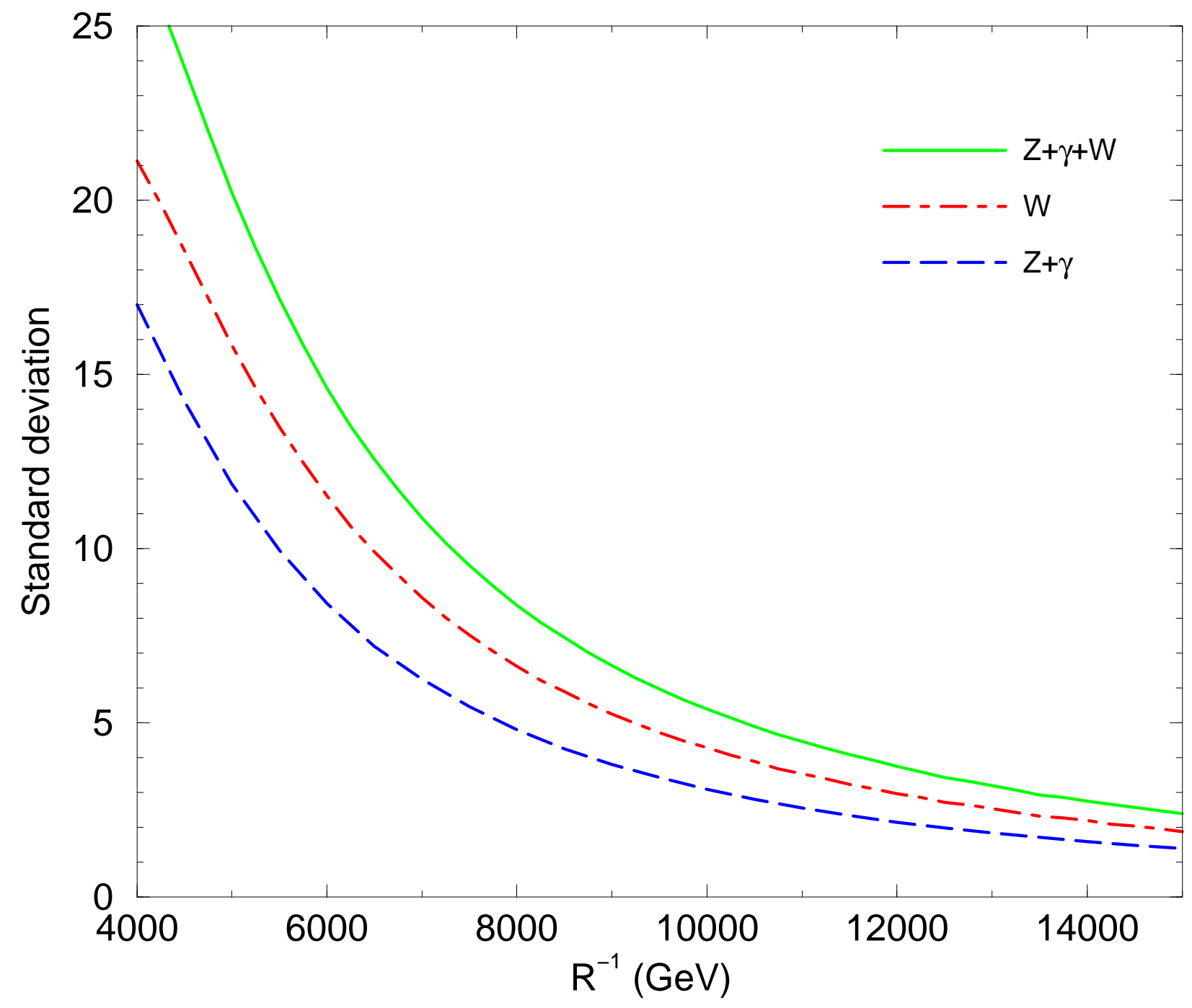

Figure 5: Number of standard deviation in the number of $l^{+} l^{-}$pairs and $\nu_{l} l$ pairs produced from the expected standard model value due to the presence of one extra-dimension of radius $R$. 


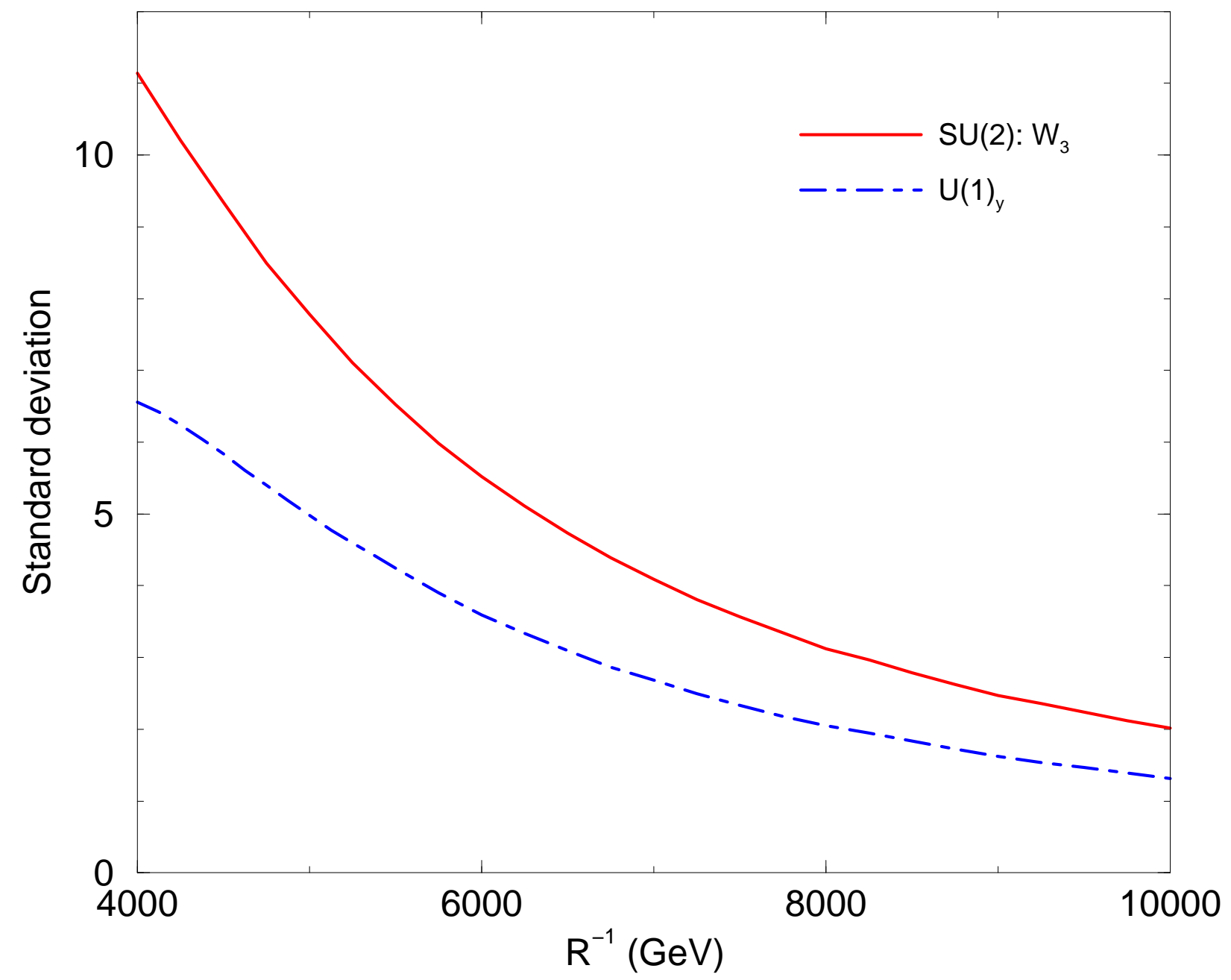

Figure 6: Number of standard deviation in the number of $l^{+} l^{-}$pairs produced from the expected standard model value due to the presence of one extra-dimension of radius $R$ in the case of $(t, l, t)$ i.e. $W_{3} K K$ excitations and the case of $(t, t, l)$ i.e. $U(1)_{Y}$ boson $K K$ excitations. 